\title{
Convivencia en espacios de racismo institucionalizado y/o político
}

Living together in areas of institutionalized racism

\author{
Iraide Fernández Aragón \\ Universidad del País Vasco/Euskal Herriko Unibertsitatea \\ iraide.fernandez@ehu.eus (ESPAÑA) \\ Julia Shershneva \\ Universidad del País Vasco/Euskal Herriko Unibertsitatea \\ Yulia.shershneva@ehu.eus (ESPAÑA)
}

Recibido: 31.10 .2016

Aceptado: 08.05.2017

\section{RESUMEN}

Las actitudes racistas, ya sean procedentes del colectivo mayoritario o dentro del propio colectivo minoritario, no surgen de manera espontánea. Como casi todo en el ámbito social, la formación de actitudes colectivas conlleva un proceso en el que dichas actitudes se manifiestan de diversas formas y en distintas intensidades.

En dicho proceso, las instituciones juegan un papel fundamental en la formación de la opinión pública y por tanto en la cohesión social, pudiendo funcionar como un instrumento de contención o bien de difusión del racismo. La diferencia entre una posición u otra marca la diferencia entre un racismo débil y que no supera los límites discursivos y otro legitimado cuyas consecuencias para la convivencia y la propia seguridad de las personas pueden ser devastadores.

En contraste con la teoría elaborada por Michel Wieviorka en su libro El espacio del racismo, trataremos de desentrañar los sucesos que comenzaron en noviembre de 2014 en la ciudad de Vitoria-Gasteiz tratando de identificar las diferentes fases descritas por el autor mediante el análisis de ocho grupos de discusión realizados en la capital vasca.

\section{PALABRAS CLAVE}

Racismo institucionalizado, cohesión social, estereotipos, inmigración. 


\begin{abstract}
A racist attitude, either from the majority group or within the minority group, does not arise spontaneously. Like almost everything in the social field, the formation of group attitudes involves a process in which these attitudes are externalized by different forms and have different strength.

In that process, the institutions play a key role in shaping of public opinion and, therefore, in social cohesion. In addition, the institutions can be the instrument of containment or transmission of racism. The difference between this two roles consist in the fact that the first one involves a weak racism and the second one legitimates discourse which consequences for coexistence and security of the people can be devastating.

According to the theory developed by Michel Wieviorka in his book The arena of racism, we try to analyze the sequence of incidents that started in November 2014 in the city of Vitoria-Gasteiz. In this respect, we try to identify the different levels described by the author by analyzing eight focus groups conducted in the Basque capital.
\end{abstract}

\title{
KEY WORDS
}

Institutionalized racism, social cohesion, stereotypes, immigration.

"El Estado está necesariamente involucrado cuando surge la violencia y es responsable, por la respuesta que le aporta, de su extensión o regresión" (Wieviorka, 2009: 89).

\section{INTRODUCCIÓN}

El racismo ha suscitado el interés de la sociología desde sus inicios, aunque fue tras los trágicos sucesos ocurridos en la Segunda Guerra Mundial cuando este tópico de estudio llegó a su apogeo. Desde ese momento, el propio concepto de racismo ha ido evolucionando y modificándose hasta nuestros días, en los que al hablar de racismo no siempre lo hacemos en términos "raza", hecho este que complejiza su interpretación.

De hecho, al pensar en racismo tendemos a imaginar un grupo dominante que manifiesta su hostilidad hacia un grupo minoritario, es decir, habitualmente las minorías étnicas se consideraban víctimas y casi nunca emisores del prejuicio. Sin embargo, hay cada vez más casos en los que se comprueba que las relaciones entre grupos minoritarios no siempre son de empatía y solidaridad. Se ha tomado como ejemplo para este artículo el caso de las acusaciones a ciertos 
grupos de inmigrantes de abusar del sistema de protección social, declaradas en Vitoria-Gasteiz (Araba) desde el nivel institucional en julio de 2014, que han puesto de manifiesto una estructura de creencias racistas dentro del propio colectivo inmigrante.

En este artículo se pretende analizar la incidencia del discurso político dentro del propio colectivo inmigrante, mostrando especial interés en las relaciones entre lo que más adelante caracterizaremos como los inmigrantes "buenos" y "malos", así como su influencia en la convivencia. Con este objetivo trataremos, en primer lugar, de diseñar un marco conceptual desde el que interpretar esta realidad.

A través del análisis de fuentes secundarias y el análisis de discurso de 8 grupos de discusión realizados en el marco del estudio "Estudio diagnóstico sobre el fenómeno migratorio en Vitoria-Gasteiz 2014" llevado a cabo en la localidad entre 2014-2015 y financiado por el Ayuntamiento del municipio, trataremos de comprobar una serie de hipótesis acerca de este fenómeno:

1. Las personas inmigrantes son actores activos del racismo, que intervienen en todas las etapas del mismo, siendo copartícipes de los mecanismos de violencia simbólica ejercida desde las instituciones.

2. Esto se ha manifestado con la intensificación del racismo y su paso a la fase institucional, que ha legitimado su expresión y ha evidenciado prejuicios y estereotipos tanto entre la población autóctona como entre la inmigrante.

Se trata, por tanto, de un intento de aplicar los planteamientos teóricos existentes sobre el racismo en las sociedades modernas a un caso, como es el de Vitoria-Gasteiz, incluyendo una perspectiva novedosa al considerar a la población inmigrante como emisora del racismo y no únicamente como su receptora. Este caso suscita un gran interés científico debido a los acontecimientos ocurridos en julio de 2014 en el municipio y, sobre todo, la radicalización del discurso político, situaciones ambas que han generado una cierta tensión social con eco mediático.

\section{LA CUESTIÓN DEL OTRO DESDE LA TEORÍA DEL RACISMO}

El racismo en la actualidad ha sufrido una transformación importante, tanto en su contenido como en las formas en las que se manifiesta. La raza ${ }^{1}$ como elemento esencial de la ideología racista ha dado paso a otras categorías y construcciones sociales, como pueden ser etnia o incluso clase social. Sin

\footnotetext{
${ }^{1}$ En este trabajo, al usar el término de "raza" o "racial", nos referiremos siempre a un constructo social y nunca a las supuestas diferencias físicas o una clasificación biológica de seres humanos. Asimismo, al hablar de racismo, se menciona en su forma más bien cultural, así llamado racismo sin raza, cuando la inferioridad es entendida como un rasgo inherente e innato de la cultura.
} 
embargo, a pesar de los avances en la lucha por la igualdad y la desaprobación social del comportamiento y discurso racista, este fenómeno no ha dejado de existir, igual que la estratificación socio-etno-racial que, según múltiples informes, persiste en las sociedades contemporáneas.

Desde que se formuló por primera vez, la teoría del racismo ha sido objeto de interés de muchos científicos, tanto del campo de Ciencias Sociales como de Biología, Medicina y otras disciplinas. En el continente europeo la teoría racista se remonta al siglo XVIII, coincidiendo con la colonización y el desarrollo del nacionalismo, y llega a su apogeo en la Segunda Guerra Mundial, cuando la ideología de existencia de "razas" humanas superiores e inferiores resultó en exterminio y matanzas colectivas de los segundos.

Tras las atrocidades ocurridas en la Segunda Guerra Mundial, así como el Apartheid en Sudáfrica y otros acontecimientos históricos basados en el odio hacia un grupo social determinado, las sociedades occidentales han elaborado mecanismos relativamente eficaces de prevención para que el racismo cotidiano no rebase esa línea que lo separa de cualquier forma de violencia física. En este sentido, los avances en derechos civiles han contribuido a que hoy en día sea menos habitual el racismo biológico, es decir, basado en las supuestas diferencias raciales como tales (Jones, 1972), y que el contenido racista se sustituya cada vez más por la jerarquización de atributos culturales, como, por ejemplo, religión, costumbres, lengua, etc. Así, Baker (1981) habla de nиevo racismo que se basa en la supuesta pérdida de identidad cultural debido al aumento de la diversidad etnoracial, mientras que otros autores definen las nuevas formas de racismo como racismo cultural centrado en mayor medida en el discurso diferencialista (Taguieff, 1988; D’Appollonia, 1998). Por otra parte, desde la psicología social se propone la utilización de los términos de racismo moderno definido como el resultado de una contradicción entre los valores igualitarios y los sentimientos negativos que suscita el otro (McConahay, 1986) o el prejuicio sutil (Pettigrew y Meertens, 1995), basado en la percepción de amenaza generada por el otro y la exageración de las diferencias culturales.

Gaertner y Dovidio (1986), a su vez, mencionan el racismo aversivo que se da cuando existe un conflicto entre valores igualitarios y sentimientos negativos hacia el otro.

Por último, Kinder y Sears plantean el concepto de racismo simbólico que consiste en la consideración de que el otro viola los valores tradicionales de la sociedad receptora (Kinder y Sears, 1981).

A pesar de la aparición de estas nuevas formas de racismo, y aunque rara vez se pretenda eliminar físicamente a un colectivo o grupo social estigmatizado, los mecanismos sociales que se despliegan para "defenderse" del otro siguen siendo similares, y tienen como resultado evitar la interacción cotidiana, estigmatizar e incluso aislar a los otros negativos.

Pero, ¿quién es ese otro y qué le caracteriza? En la literatura sociológica hay muchas formas de referirse a la figura del otro, extraño o monstruo. Básicamente, la mayoría de los autores coinciden en que son aquellos que potencialmente pueden quebrar el orden social e introducir caos en él. Así son 
las figuras del forastero y extranjero de Alfred Schütz (1944) y Georg Simmel (1977), o la del extraño de Ulrich Beck (2000). Son seres anómicos, que pueden incluso llegar a ser considerados como impuros y contaminantes en el imaginario social (Douglas, 1973) ${ }^{2}$. Todas ellas son manifestaciones de la otredad, en mayor o menor medida, pero que al fin y al cabo tienen que ser "neutralizados", si no eliminados del espacio simbólico y/o material.

Uno de los mecanismos racializantes es el racismo estructural o institucional $^{3}$, descrito por primera vez por Carmichael y Hamilton (1967) y posteriormente desarrollado por otros autores (Wieviorka, 1992), que son prácticas que, sin tener una base racista o actores claramente definidos, perpetúan la situación de desventaja de los grupos sociales desfavorecidos, manteniéndolos en la posición subordinada y de desventaja. Por tanto, el racismo institucional está incrustado en las prácticas sociales de una sociedad de tal forma que muchas veces pasa desapercibido, manifestándose en diversos mecanismos sociales.

\section{CUANDO EL RACISMO DETERMINA LAS RELACIONES SOCIALES}

Ahora bien, ¿cuándo el racismo latente se transforma en violencia física y otras formas de hostilidad más extremas? Son varios los autores que se han preocupado por establecer relación entre el prejuicio y la acción. La conclusión a la que se llega es que normalmente esta relación no es automática y directa, sino que se tiene que dar una serie de condiciones favorables previas, siendo una de estas condiciones la legitimación a desde el nivel político e institucional del discurso xenófobo y racista.

Así, uno de los primeros autores en ofrecer una clasificación de estadios por los que pasa el racismo y la xenofobia antes de llegar a su manifestación extrema ha sido Gordon Allport (1962). El autor distingue cinco manifestaciones que varían en su grado de intensidad y, por consiguiente, sus consecuencias. La forma más común y menos intensa en la que se expresan las actitudes racistas y xenófobas es la verbal. El segundo grado de manifestación es la evitación del contacto con el otro, mientras que en el tercer estadio se caracteriza por la discriminación del grupo que se quiere evitar, negándole derechos y privándolo del poder y, por tanto, de posibilidad de cambiar su situación. El ataque físico y el exterminio en su forma extrema tendrían lugar en el quinto nivel y tendría consecuencias muy graves para el grupo víctima de dicha agresión.

En esta misma línea, Wieviorka (1992/2009) hace una propuesta teórica de clasificación de etapas o planos del racismo, que son los procesos sociales que preceden la violencia racista propiamente dicha, identificando los siguientes

${ }^{2}$ Para una revisión más detallada consultar Izaola y Zubero (2015).

${ }^{3}$ No debe confundirse el racismo institucional o estructural con el ejercido desde las élites políticas caracterizado por algunos autores como racismo institucionalizado. 
espacios de racismo: infrarracismo, racismo disperso, racismo institucionalizado y, por último, racismo total.

La primera etapa, llamada infrarracismo, que precede a la violencia física es la construcción del otro negativo, que consiste en despliegue de diversas prácticas sociales que preparan el espacio simbólico para deslegitimar a aquellos que encarnan lo extraño, es decir, aquello que debe estar fuera de la sociedad. Los estereotipos y prejuicios, ya sean a base de "raza", etnia, religión o cultura, fomentan un clima social de desconfianza y temor hacia este otro negativo constituyendo el caldo de cultivo para el desarrollo del racismo.

En la segunda fase, denominada racismo disperso, es cuando estos prejuicios y estereotipos empiezan a coger cuerpo y resultan en una tensión social, que se traduce en la segregación espacial y evitación de los grupos estigmatizados, así como de violencia racista puntual y localizada protagonizada por grupos de extrema derecha aislados. Asimismo, el miedo al otro y la percepción de amenaza que éste encarna llegan a un nivel muy elevado.

La barrera que separa esta fase de la posterior es la legitimación desde las instituciones que sustentan el poder del discurso en torno a la inmigración. En este sentido, la postura institucional puede desempeñar el rol de una valla de contención del odio o abrir espacios de impunidad y violencia física y eliminación simbólica del otro.

La fase posterior, racismo político, a su vez, emerge cuando se involucran las élites políticas propiciando la discriminación y la segregación, ya sea de forma directa o indirecta. Los partidos políticos de derechas se activan, incluyen el discurso racista en sus programas electorales y orientan la opinión pública hacia posicionamientos racistas.

Por último, en la cuarta fase, del racismo total, es cuando el Estado se reorganiza bajo presupuestos racistas, integrándolo en todas las esferas de la vida pública y legitimando la ideología racista. Es decir, la violencia es ejercida y organizada desde el nivel político. Esta fase es la que más peligro supone, ya que en la práctica puede llevar a consecuencias muy trágicas como sucedió, por ejemplo, en Sudáfrica o la Alemania nazi.

Aunque el paso de una a otra fase a veces no es fácil de determinar, la diferencia más pronunciada está en esa línea de la institucionalización que separa las dos primeras de las dos últimas, ya que, una vez cruzada, transforma el racismo en una ideología especialmente peligrosa, pues empieza a tener la capacidad de organización y contar con otro tipo de recursos. En otras palabras, cuando el racismo es legitimado desde arriba, se empodera y puede resultar en violencia.

En el siguiente esquema se ofrece un resumen del proceso que atraviesa el racismo antes de llegar a la violencia física sistematizada (Esquema 1). Así, cada nivel supone una mayor complejidad, pero también una radicalización de las posturas y actitudes socio-políticas. 


\section{Esquema 1. Etapas del racismo: de prejuicios a violencia física}

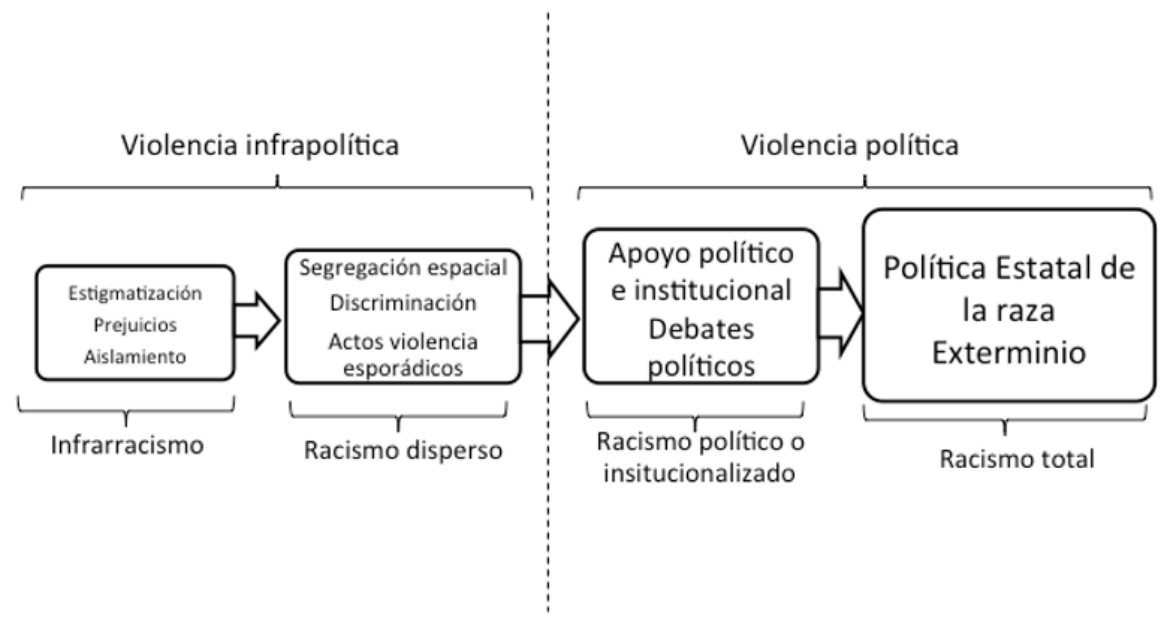

Fuente: Elaboración propia

Respecto a las teorías explicativas, que tratan de encontrar las causas del racismo, podemos diferenciar entre aquellos enfoques que hacen hincapié en el contexto, aquellos que lo hacen desde el ámbito de la personalidad y, por último, aquellos que lo hacen desde el grupo y las relaciones intergrupales. Entre las más comunes se encuentran las grupales, especialmente la teoría del conflicto realista (Sherif, 1966), que explica el surgimiento de la hostilidad intergrupal al percibir que los intereses de los grupos entrar en conflicto, es decir, en una situación de competencia por recursos limitados. No es casualidad que el prejuicio racista y xenófobo aflore en contextos de máxima escasez como las crisis económicas, donde se percibe que el grupo minoritario se apropia de los escasos recursos (Cea, Valles y Eseverri, 2013). También entre las teorías grupales, encontramos la denominada teoría de la identidad social (Tajfel, 1981; Tajfel y Turner, 1986), que parte de la premisa de que para mejorar la percepción de identidad social positiva, los grupos e individuos establecen comparaciones intergrupales "hacia abajo", es decir, basadas en la posición social relativa del grupo.

Hasta el momento la inmensa mayoría de las teorías parten de la hipótesis de que es el grupo mayoritario el emisor de racismo, sin contemplar la posibilidad de los grupos minoritarios también puedan sumarse a estas lógicas racializantes y discriminatorias dirigidas hacia otras minorías más vulnerables. Así, las pocas investigaciones que plantean que el racismo pueda ser protagonizado por los propios grupos minoritarios pertenecen al campo de la psicología social (Shapiro y Neuberg, 2008; Chen, 2010; Cuadrado et al., 2016). Es ésta la perspectiva que se incluye en el presente artículo, como medio para demostrar la capacidad del discurso de las élites políticas de penetrar en la sociedad en su conjunto, 
incluyendo los grupos minoritarios.

\section{DISEÑO METODOLÓGICO}

Para este análisis, nos apoyaremos en datos de fuentes secundarias y en análisis de discurso de una serie de grupos de discusión realizados en el marco del estudio "Estudio diagnóstico sobre el fenómeno migratorio en VitoriaGasteiz 2014" realizado en la localidad durante el 2014. Este municipio ha sido seleccionado por su interés especial para esta investigación, ya que los datos recientes revelan el endurecimiento del discurso y las acciones de su población autóctona hacia la población inmigrante, así como una radicalización del discurso político, situaciones ambas que han generado una cierta tensión social con eco mediático que ha provocado multitud de respuestas de diversos colectivos sociales de la localidad, incluidos los propios inmigrantes.

En lo referente a la metodología cualitativa, el estudio de las actitudes hacia la inmigración presenta una serie de dificultades debido a la elevada deseabilidad social que hace compleja su medición a través de indicadores directos (por ejemplo, encuesta) (Cea D'Ancona, 2009). En este estudio se opta por los grupos de discusión, como metodología cualitativa, porque permite profundizar en el discurso y establecer relaciones contextualizadas, de compleja detección en el análisis cuantitativo. Asimismo, el contexto polémico que genera la discusión permite obtener argumentación respecto a los posicionamientos (Gimeno, 2001). De hecho, esta misma técnica se ha empleado con exitoso resultado en proyectos como MEXEES I (2006/2008) y MEXEES II (2010/2012) .

El trabajo de campo del "Estudio diagnóstico sobre el fenómeno migratorio en Vitoria-Gasteiz 2014" se desarrolló entre el 23 de octubre y el 1 de noviembre de 2014, que coincidió con la polémica que se había generado en el municipio en torno al supuesto abuso de las prestaciones sociales por colectivos de inmigrantes concretos, acusándolos, además, de la incapacidad de integración en la sociedad vasca. Esta circunstancia hace que el contexto estudiado sea de especial interés para nuestro objetivo, ya que permite determinar la respuesta de la sociedad, y en especial de la propia población inmigrante, ante esta radicalización del discurso político.

Concretamente se han realizado ocho grupos de discusión formados por personas de origen extranjero de ambos sexos mayores de 17 años, residentes en Vitoria-Gasteiz y de las principales áreas de procedencia del municipio:

${ }^{4}$ Financiado por el Ayuntamiento del municipio.

${ }_{5}^{5}$ El proyecto MEXEES I (La medición de la xenofobia en la España de comienzos del siglo XXI: modelos para la implementación de políticas de integración social) bajo la dirección de María Ángeles Cea D'Ancona en 2006-2008; el proyecto MEXEES II (La medición de la xenofobia en la España de comienzos del siglo XXI: nuevos indicadores y diseños de encuesta para las políticas de integración social de los inmigrantes) se realizó bajo la dirección de María Ángeles Cea D'Ancona en 2010-2012. 
Grupo 1 (MA): formado por once mujeres de origen magrebí, la mitad de ellas empadronadas hasta 2008 y la otra mitad de 2009 en adelante;

Grupo 2 (MO): formado por seis hombres de origen magrebí, la mitad de ellos empadronados hasta 2008 y la otra mitad de 2009 en adelante;

Grupo 3 (LA): formado por nueve mujeres de origen latinoamericano, la mitad de ellas empadronadas hasta 2008 y la otra mitad de 2009 en adelante;

Grupo 4 (LO): formado por diez hombres de origen latinoamericano, la mitad de ellos empadronados hasta 2008 y la otra mitad de 2009 en adelante;

Grupo 5 (RA): formado por nueve mujeres de origen de Europa del este, la mitad de ellas empadronadas hasta 2008 y la otra mitad de 2009 en adelante;

Grupo 6 (RO): formado por cinco hombres de origen de Europa del este, la mitad de ellos empadronados hasta 2008 y la otra mitad de 2009 en adelante;

Grupo 7 (AA): formado por diez mujeres de origen subsahariano, con diferentes años de empadronamiento en el municipio de Vitoria-Gasteiz;

Grupo 8 (AO): formado por once hombres de origen subsahariano, con diferentes años de empadronamiento en el municipio de Vitoria-Gasteiz.

Se ha intentado garantizar la homogeneidad interna de cada grupo en cuanto a las variables origen y sexo, con el objetivo de reducir el sesgo de deseabilidad social a la hora de expresar el discurso actitudinal sobre otros colectivos.

Las sesiones, de una duración aproximada de dos horas, se dinamizaron utilizando un guión que contenía 8 dimensiones que recogieran la realidad migratoria del municipio: proceso migratorio, entorno residencial, convivencia integración, ámbito laboral, ámbito educativo, esfera privada, expectativas e imagen del Ayuntamiento.

Respecto a las fuentes secundarias, con el fin de contextualizar el discurso recabado en el campo cualitativo, se han utilizado los datos del Padrón municipal de habitantes de Vitoria-Gasteiz del Instituto Nacional de Estadística (INE).

\section{CONTEXTO MIGRATORIO DE VITORIA-GASTEIZ}

El fenómeno migratorio internacional es una cuestión relevante para la ciudad de Vitoria-Gasteiz, donde residen 20.881 personas de nacionalidad extranjera a 1 de enero de 2016, lo que supone el 8,5\% del total de la población del municipio y el $81,7 \%$ de la población residente en la provincia alavesa (Tabla 1). De hecho, a pesar de que la Comunidad Autónoma de Euskadi (en adelante CAE) presenta porcentajes de personas extranjeras por debajo de la media estatal, que asciende a 9,9\%, Vitoria-Gasteiz muestra un volumen más cercano a esta media. 
Tabla 1. Población por nacionalidad y territorio, absolutos y \% horizontales, (2016)

\begin{tabular}{|l|l|l|l|l|}
\hline \multirow{2}{*}{} & \multicolumn{2}{|l|}{ Nac. española } & \multicolumn{2}{l|}{ Nac. extranjera } \\
\cline { 2 - 5 } & N & \% & N & \% \\
\hline España & 41.938 .427 & 90,1 & 4.618 .581 & 9,9 \\
\hline CAE & 2.050 .109 & 93,6 & 139.425 & 6,4 \\
\hline Araba & 298.579 & 92,1 & 25.547 & 7,9 \\
\hline Vitoria-Gasteiz & 223.753 & 91,5 & 20.881 & 8,5 \\
\hline
\end{tabular}

Fuente: Elaboración propia. INE

En lo referente a la distribución de orígenes en la capital alavesa, se observa en la Tabla 2 que los orígenes africanos suponen un 43,7\% de la población extranjera residente en el municipio, siendo Magreb quien detenta un mayor protagonismo. De hecho, la presencia de población africana es mucho más marcada en la capital que en la CAE, donde suponen un $18,8 \%$ del total de población extranjera (INE, 2016). También las personas procedentes de Latinoamérica suponen un $26,3 \%$, aunque es un porcentaje relativamente bajo si lo comparamos con el 32,8\% para la CAE. Por sexo, se observan diferencias reseñables, siendo Latinoamérica el origen más feminizado y Asia y África Subsahariana los más masculinizados.

Tabla 2. Población de nacionalidad extranjera por áreas geográficas y sexo, \% verticales y horizontales (2016)

\begin{tabular}{|l|l|l|l|}
\hline & Hombres & Mujeres & $\begin{array}{l}\text { To t a I. \% } \\
\text { vertical }\end{array}$ \\
\hline África Subsahariana & 57,2 & 42,8 & 17,4 \\
\hline Magreb & 56,3 & 43,7 & 26,3 \\
\hline Resto UE & 57,8 & 42,2 & 7,3 \\
\hline Rumanía y Bulgaria & 50,3 & 49,7 & 6,4 \\
\hline Europa no comunitaria & 44,2 & 55,8 & 5,2 \\
\hline América Latina & 40,8 & 59,2 & 26,3 \\
\hline Asia & 64,5 & 35,5 & 11,1 \\
\hline Total & 52,4 & 47,6 & 100 \\
\hline
\end{tabular}

Fuente: Elaboración propia. INE 
Se observa, por tanto, que Vitoria-Gasteiz tiene un perfil migratorio diferencial, fruto de las características específicas de su mercado laboral, centrado en el sector de agricultura y construcción, muy castigados por la crisis económica que comenzó 2008. Esto en parte explica la vulnerabilidad de la población de origen africano en términos sociales y económicos, ya que este colectivo se empleaba fundamentalmente en estos sectores (Ikuspegi, 2014).

Respecto a la distribución del colectivo inmigrante en el espacio urbano, las personas de nacionalidad extranjera no se concentran en un mismo barrio, aunque más del $50 \%$ reside en 7 barrios de la capital. Zabalgana es el barrio en el que más personas de nacionalidad extranjera viven, el 10,1\%, seguido de Coronación en el que residen el 8,7\%. Lakua, la zona de más reciente construcción, concentra el 7,3\% de este colectivo. Como ocurre en muchas ciudades, también la parte más antigua de la ciudad, como el Casco viejo, es destino para el parte de este colectivo (Ikuspegi, 2014). Es decir, a pesar de que existen algunas zonas donde la población extranjera está sobrerrepresentada, no podemos hablar de segregación residencial.

Por último, cabe destacar para el caso de Vitoria-Gasteiz el ámbito educativo (Tabla 3), ya que supone una característica singular respecto a otros municipios. En primer lugar, en la CAE existen tres modelos lingüísticos impartidos en los centros educativos: modelo A, en el que predominan las asignaturas en castellano; modelo B, castellano y euskera; y modelo $\mathrm{D}$, donde predominan las asignaturas en euskera. Si bien desde hace años a población autóctona se matricula mayoritariamente en modelos que se cursan en euskera, la población extranjera residente en la capital alavesa presenta una tendencia mayoritaria a hacerlo en modelos impartidos en castellano (61,3\%), tendencia más acusada que para el caso de la CAE (51\%) (Ikuspegi, 2014). Además, la existencia de dos redes educativas, la pública y la concertada (de carácter privado pero financiada parcialmente públicamente), establece también diferencias importantes, siendo Vitoria-Gasteiz la capital con un mayor porcentaje de alumnado extranjero matriculado en la red pública (el $81 \%$ ) en comparación con la CAE (75\%) (Ikuspegi, 2014).

Tabla 3. Alumnado inmigrante por modelo y red escolar, Vitoria-Gasteiz, \% horizontales y absolutos, (2014)

\begin{tabular}{|l|l|l|l|l|l|}
\hline & $\begin{array}{l}\text { N } \\
\text { alumnado } \\
\text { extranjero }\end{array}$ & A & B & D & Total \\
\hline Público & 5.141 & 58,7 & 18,3 & 23 & 100 \\
\hline Concertado & 1.131 & 73,0 & 25,7 & 1,2 & 100 \\
\hline Total & 6.272 & 61,3 & 19,7 & 19,1 & 100 \\
\hline
\end{tabular}

Fuente: Elaboración propia. Gobierno Vasco 
En segundo lugar, y en relación con la distribución de alumnado inmigrante en los centros de la capital, tal y como se apunta en el citado informe de 2014, la concentración espacial de personas extranjeras en determinados barrios, unida a la tendencia a matricular en la red pública y modelo A, genera una cierta segregación escolar con centros en los que se sobrepasa el 30\% de alumnado extranjero, porcentaje deseable establecido por el Gobierno Vasco (Ikuspegi, 2014).

\section{RESULTADOS}

\subsection{Espacios de racismo: el caso de Vitoria-Gasteiz}

En este apartado, trataremos de relacionar las diferentes etapas caracterizadas en el apartado anterior con los sucesos y procesos ocurridos entre los años 2014 y 2015 en el municipio de Vitoria-Gasteiz.

\subsubsection{Construyendo el otro negativo}

Como se ha podido observar en el primer apartado, el racismo, antes de adquirir sus formas extremas, pasa por una fase preliminar, que podríamos caracterizar como un racismo débil o de fuerza baja, que no es organizado ni sistemático. Se manifiesta mayoritariamente de manera discursiva y sólo en casos aislados de manera física, que normalmente no guarda relación con la postura política. Es en esta etapa donde empieza a forjarse la figura del otro negativo, que puede ser trazado en los discursos de la gente, pero que únicamente aparecen de forma general y sutil. Este otro no evoca simpatía, pero tampoco es estigmatizado de forma efectiva o perceptible para él. Estaríamos ante unas manifestaciones de racismo y xenofobia moderadas que no dan lugar a comportamiento diferenciador y aislante respecto a este otro.

En el caso que se analiza, a este nivel corresponderían las actitudes xenófobas y racistas moderadas que forman parte de la realidad vitoriana, no sólo en el imaginario de la población autóctona, sino también entre el propio colectivo inmigrante que dista de ser homogéneo y maneja también sus propios estereotipos, en ocasiones similares a los de la población autóctona. Para ilustrarlo, se aportan algunos de los comentarios que recabados en la presente investigación:

Entre nosotros también podemos ser racistas, porque conocí a una cubana que siempre me llamaba negra de manera despectiva. $A A$

[Los africanos] no quieren integrarse. LA

Los gitanos y moros llevando navajas [en el centro escolar]con trece años. $L O$ 


\section{[Los marroquíes] Son sucios. LA}

Sin contar cómo [los marroquíes] desprecian a la mujer. LA

[Los marroquíes] te roban. LA

Normalmente los problemas de convivencia suelen ser con los árabes. LA

¿La droga quién la vende? Ver a un moro trabajando, lo firmo. LA

[Los africanos]Ni siquiera aprenden el idioma. Es que los ves y es que ni siquiera saben hablar aunque lleven toda la vida. LA

Yo cuando vine aquí la primera vez a Vitoria, siempre era "que los moros, que los moros". Y pensé "iqué racistas son los vascos con los moros!". Pero después me di cuenta conviviendo con ellos y queriendo trabajar con ellos de que realmente da bronca, pero es que tienen mucha facilidad, más que cualquiera. Más que cualquier español o que cualquier latino. $L A$

En este punto comienza ya a cristalizarse la figura del otro negativo, visto desde la mirada del inmigrante: el "moro". Aparece de forma recurrente, sobre todo, en el discurso de la población latinoamericana y rumana que, además, ocupan una posición privilegiada en el imaginario de la población autóctona (Cea D'Ancona y Valles, 2014; Ikuspegi, 2015). Estas actitudes racistas y xenófobas hacia el colectivo magrebí se detectan no solo en el caso de VitoriaGasteiz, sino también a nivel nacional (Cea D'Ancona y Valles, 2014) e incluso, como tendencia internacional. El aislamiento, que se que se da en la etapa que se describe a continuación, es consecuencia de los prejuicios que se generan en este nivel de infrarracismo, que actúan como contexto discursivo.

\subsection{Cuando las creencias se vuelven tangibles: "No quiero nada de contacto con ellos"}

Tras el primer nivel, en la siguiente fase se aprecian ya las manifestaciones físicas o "tangibles" de las actitudes racistas y xenófobas presentes en la sociedad. Donde la evitación del contacto comienza a manifestarse residencialmente y la interacción con el grupo estigmatizado disminuye.

Aunque para el caso de la CAE no se encuentran casos extremos de segregación urbana ${ }^{6}$, -entendida esta como la distribución de diferentes colectivos en el espacio urbano de manera sobrerrepresentada-, se encuentra una cierta presencia de personas de origen extranjero en determinadas zonas

${ }^{6}$ Este fenómeno supone un tópico de estudio para la sociología desde los años 30, en el contexto de la escuela de Chicago, donde se convirtió en una rama de gran importancia bajo su máxima "Distancia espacial equivale a distancia social" (Park, 1967). A pesar de la frecuencia con la que leemos o escuchamos la palabra gueto, esta categoría hace referencia únicamente a aquellas situaciones de segregación extrema en las que el 100\% de los residentes en un área pertenecen al mismo colectivo. 
de la ciudad ${ }^{7}$, que además queda reflejada en los testimonios de las personas inmigrantes.

Dichos procesos de diferenciación socio-residencial se explican en parte por la preferencia de los miembros de un grupo de vivir en zonas donde las personas de ese mismo grupo sean mayoritarias y, en parte, por el deseo la población autóctona de distanciarse de ciertos colectivos de inmigrantes, hecho este que se confirma en los sondeos (Ikuspegi, 2015). Esta tendencia de distanciamiento de la población mayoritaria queda reflejada en los testimonios de algunas personas entrevistadas:

[...] cuando se daban cuenta que en el piso viviríamos nosotros, inmigrantes negros, solo decían que nos llamarían, lo que nunca hacían. AO

La primera vez cuando hemos buscado piso yo y mi mujer, la mujer que nos atendía y se ha enterado de que somos rumanos, empezó temblando. No sabía cómo librarse de nosotros. No sé, igual he tenido malas experiencias. Todo el mundo tiene algo de racismo dentro, aunque no lo reconoce. RO

A mí me paso que llevaba cuatro meses buscando habitación. Un día llame a un piso y me pregunta de dónde soy a lo que contestó que de Senegal. Seguido me pregunta si soy negra y si mi hija también es negra. Y le dije claro como yo. Al final nada. AA

Como podemos observar, el racismo disperso, acompañado por la situación de desventaja socioeconómica, da lugar a consecuencias tangibles para la población inmigrante, que se traducen en la concentración de la población inmigrante en ciertos barrios, algo percibido también por este colectivo:

Hay barrios donde sólo hay inmigrantes. AA

Ahora en los barrios nuevos, Salburúa, Zabalgana, vas a encontrar gitanos, marroquíes y latinos. LO

El Casco Viejo está lleno de extranjeros. He vivido ahí un año y ya me he mudado en Badaia. RA

Yo vivo en Salburúa y parece que a todos los inmigrantes nos han metido ahí. $L A$

En mi barrio se vive como en África. Mucho de África. Ves a la gente sentada en el portal de sus casas. AO

Hay mucha gente de fuera, si es un barrio nuevo como Salburúa. RA

En nuestros barrios no hay mucha gente local, la mayoría es gente de fuera. $A A$

Bueno, donde vivo yo hay gente de todo tipo: de aquí, de Marrueco, Algeria, Colombia. RO

${ }^{7}$ Consultar contexto para mayor detalle. 
Pero es que hay guetos de viviendas. Hay una parte en Salburúa, cruzando el puente, que es de moros. Cruzas una zona y todos los portales son de moros. LA

Los gitanos [rumanos] traen problemas, pues Abetxuko es de los gitanos. Los gitanos tienen su lugar. Traen problemas, es verdad. LA

Pero en Zabalgana la concentración de extranjeros y gitanos ha hecho que la convivencia fuera algo complicada. Hay poco contacto con la gente autóctona. $M O$

Otra consecuencia de la manifestación de los prejuicios tangible la encontramos en otros ámbitos, donde el otro es evitado y aislado desde la población mayoritaria, que expresa sus actitudes negativas a través de un trato diferenciado:

Aquí por ejemplo hay restaurante, marroquíes, latinos, pero si abrimos nosotros, no iría ni dios. Porque van a empezar a interpretar nuestra comida como sucia. AA

Aunque tengas formación aquí, carrera y todo nunca te van a contratar por tu color de piel. $L O$

Yo por que tenga un color de piel más oscuro, aunque tenga mis estudios, aunque tenga un currículum de puta madre, no puedo optar a un puesto específico en un ayuntamiento porque se ve que no soy de aquí. $A A$

Los blancos por ejemplo no les dejan a los niños que se mezclen [con los de fuera]. AA

Entras en un bus y te sientas a lado de un local, se levanta y se va. AA

La concentración del alunando inmigrante en ciertos centros educativos es otro ámbito en el que se manifiesta la matriz social racista, aunque muchas veces camuflada con la desventaja económica y social. Da cuenta de la existencia de una serie de mecanismos que ubican a ciertos colectivos estigmatizados en una posición desventajosa y subordinada, los mismos mecanismos que plantea Bourdieu hablando de la violencia simbólica, que son esquemas y estrategias del ejercicio del poder implícitas empleadas desde el grupo dominante, a menudo interiorizadas y reproducidas por los propios sujetos subordinados, para mantener el orden social y la subordinación de grupos desfavorecidos (Bourdieu y Wacquant, 1992; Fernández, 2005).

Así, una de las consecuencias de estos procesos es la sobrerrepresentación de los colectivos estigmatizados en algunos centros educativos caracterizados por la falta de recursos. Así ocurre en el caso estudiado, donde existen centros públicos de la capital vasca, que superan con creces el límite recomendado desde las propias instituciones vascas (en torno a un 30\% de alumnado de origen extranjero). Puesto que la escolarización en el sistema público de enseñanza en la CAE se realiza por cercanía a la zona de residencia del alumnado, la sobrerrepresentación residencial de la inmigración se reproduce en el ámbito educativo, generando centros educativos con una alta concentración del 
alumnado inmigrante. Al mismo tiempo, la tendencia que muestra la población inmigrante de matricular a sus hijos e hijas en la red pública y en el modelo lingüístico en castellano, contribuyen a una mayor segregación escolar (consultar contexto).

Esta situación resulta evidente tanto para la población autóctona vitoriana donde un tercio de la población se muestra de acuerdo con la afirmación de que el alumnado inmigrante baja el nivel educativo (Ikuspegi, 2014), pero también observamos estas mismas cuestiones en la opinión de la propia población inmigrante, que identifica este tipo de centros y relaciona la concentración con la pérdida de calidad de los servicios:

Hay centros educativos únicamente para extranjeros y eso parece exclusión. AO

Donde hay edificios nuevos, también se hacen escuelas nuevas. Entonces, a los niños que están ahí no los vas a llevar a estudiar al centro. Tendrás que zonificarlo. Y tu niño va a ir con gente de Marruecos, con gente africana, con gitanos. Se integrará ahí. LO

[...] nos mandaron a mí y a mis hermanos a los peores colegios. MA

A los que vienen de fuera les llevan a los peores colegios donde hay peleas fuera. MA

El colegio de las bestias. LA

Cada uno viene con su lección, sus normas y no sale bien. RO

Esta situación, también de forma unánime, es valorada como un aspecto negativo que ejerce como barrera a la integración y que provoca consecuencias negativas en el alumnado. Al mismo tiempo, esta insatisfacción con una red pública que se percibe como segregada, conflictiva y de baja calidad, tiene consecuencias en algunos colectivos, como el latinoamericano o el rumano, que emplean diferentes estrategias para evitar el contacto con el otro: cambiar de residencia a otra área o la matriculación en un centro concertado:

Hemos tenido que coger y empadronarnos en casa de un amigo que vive cerca. $L O$

Les voy a comentar el caso de una amiga colombiana que ella quiso sacar a su hijo del colegio público por la inmigración, por la cultura marroquí. Ella no quiere que su hijo ande con esa gente. LO

Vienen niños de muchos países y ahí también muchos de Marrueco, de las mujeres que se ponen velo, yo no las entiendo, no quiero nada de contacto con ellas. $R O$

Yo la saqué [a mi hija] de allí y la metí en el concertado. LA

Mi hermana no quería ir a ese colegio, estaba lleno de inmigrantes. $M A$

Yo me he mudado al nuevo barrio, quiero cambiar el colegio de mi hija porque ahi el ambiente deja mucho que desear. RO 
Tengo gente que ha tenido a los hijos en castellano y venga a repetir y venga a juntarse con no sé qué y venga a robar a no sé dónde. No quiero que mi hijo esté en esta mezcla de gente. RA

Se observa que, que al igual que en el caso de la población autóctona, dentro del propio colectivo inmigrante, se emplean estrategias para no compartir espacios con aquellos colectivos estigmatizados, contribuyendo a su aislamiento, pero también determinando las futuras generaciones. Estos colectivos se suman a los procesos de violencia simbólica, aunque habitualmente no se consideran actores activos en las numerosas investigaciones sociales realizadas en torno a este fenómeno.

\subsection{Racismo de arriba a abajo}

Una vez superadas las etapas de racismo de menor intensidad y como se mencionaba anteriormente en el esquema inicial, la postura de las instituciones políticas juega un rol fundamental a la hora de legitimar o deslegitimar las actitudes racistas y xenófobas de la población. En este sentido, la cuestión migratoria, habitualmente desde la perspectiva de "culpables", se coloca en el centro del debate político Este nivel incluye el racismo indirecto (o institucional); es decir, el establecimiento de mecanismos administrativos o institucionales, así como el racismo más directo, declaraciones y/o normativas específicas para impedir la entrada de miembros de un colectivo.

Pasar de un escenario donde las instituciones se mantienen al margen o, incluso, tratan de solucionar un conflicto a otro en el que forman parte del mismo conflicto no es una cuestión baladí. En este sentido, lo ocurrido en la capital alavesa, cuando el 16 de julio de 2014, el alcalde popular del municipio realizaba una serie de declaraciones en un contexto pre-electoral. El edil afirmaba que las personas de origen marroquí y argelino abusaban de las ayudas sociales y no pretendían trabajar o integrarse. Establecía una comparación cuantitativa con las personas de origen latinoamericano para mostrar la sobrerrepresentación del colectivo magrebí en las ayudas sociales. No era la primera vez que este mismo gobierno entraba en conflicto con este colectivo, ya que unos meses antes prohibió a las mujeres de confesión musulmana bañarse en la piscina municipal con ropa. Sin embargo, las mencionadas declaraciones tuvieron un seguimiento importante en los medios de comunicación locales y nacionales generando un gran impacto y debate entre la población local ${ }^{8}$.

De alguna manera, se da luz verde a la población vitoriana para que culpabilice a las personas de origen magrebí, legitima un estereotipo ya muy presente en la sociedad y lo convierte en el chivo expiatorio en un contexto de

${ }^{8}$ Existe gran variedad de noticias sobre estas declaraciones que pueden consultarse. Se adjunta a modo de ejemplo: "El alcalde de Vitoria acusa a marroquíes y argelinos de vivir de las ayudas y no querer trabajar" (http://www.elmundo.es/pais-vasco/2014/07/15/53c5594aca474194 $358 \mathrm{~b} 458 \mathrm{c} . \mathrm{html})$. 
crisis económica. De hecho, ante esta situación la población autóctona tiende a sentirse desprotegida por las propias instituciones públicas en detrimento del colectivo inmigrante. Así se refleja en el último informe del Gabinete de Prospecciones Sociológicas para la CAE, en el que el 63\% de la población vasca considera que las personas inmigrantes son el colectivo social más protegido frente a las personas jóvenes, las pensionistas, etc. (Gobierno Vasco, 2012). El discurso de las élites políticas, otorga veracidad a un hecho, sin explicar el contexto en el que se enmarca y da permiso a su población para dirigir su odio hacia todo un colectivo estigmatizado. El que ya fuera el otro negativo, queda marcado y señalado desde las propias instituciones.

Por supuesto, este fenómeno de gran magnitud mediática generó rápidamente consecuencias sociales en un entorno que ya estaba predispuesto para culpabilizar a este colectivo y que sólo requería "permiso". Las respuestas a este hecho fueron diversas en función del colectivo social. Sin embargo, los mecanismo de protección presentes en las sociedades democráticas que se han mencionado en el apartado anterior, generan diversidad de opiniones, fruto de la sensibilización de la sociedad. Los propios partidos de la oposición, de manera unánime se manifestaron en contra de dichas declaraciones considerándolas xenófobas y peligrosas. La reacción de propio alcalde antes acusaciones, fue reafirmarse y, la del Partido Popular, mostrarle su apoyo.

Socialmente, podemos diferenciar dos posiciones ante las declaraciones en las que se ubicaron distintas organizaciones del tejido asociativo local: en contra y a favor. Multitud de organizaciones y personalidades se mostraron en contra de estas declaraciones que consideraron xenófobas. Una de las más mediáticas fue SOS Racismo Álava, que llevó el caso a la Audiencia Provincial por "incitar al odio". Se generó también una iniciativa popular denominada "Gora Gasteiz"10 que rechazaba las declaraciones del alcalde por el daño infligido a la convivencia en la capital.

Sin embargo, el mensaje negativo lanzado desde las instituciones fue recogido y trasmitido por amplios sectores de la ciudadanía y el tejido asociativo. En su discurso podemos apreciar una clara distinción entre inmigrantes "malos" y "buenos", discurso que no es azaroso, ya que recoge las simpatías y antipatías previas de la población en su conjunto hacia determinados colectivos. Esta distinción, lejos de pasar desapercibida, se convierte en munición para unos y losa para otros dentro del propio colectivo inmigrante. Así lo demuestra la iniciativa "Ayudas + Justas"11, una plataforma ciudadana que, tras las declaraciones, surge con el objetivo de evitar el fraude y abusos de las ayudas sociales e incluir a las personas que se quedan fuera.

En este sentido, es destacable la reacción de algunas asociaciones latinoamericanas que apoyaron e impulsaron la difusión de estos argumentos

${ }^{9}$ Más tarde la Superior del País Vasco archivaba la denuncia contra el alcalde por considerar que realizó esas declaraciones en el ejercicio de su libertad de expresión.

${ }^{10} \mathrm{http}: / /$ goragasteiz.com

${ }^{11}$ http://www.ayudasmasjustas.org/ 
que buscaban asentar la figura del otro negativo, pero también diferenciarse. Esta conducta, muestra coherencia con la mencionada teoría de la identidad social (Tajfel, 1981; Tajfel y Turner, 1986), donde tras identificar un grupo socialmente peor posicionado, se realiza una comparación intergrupal con dicho grupo para reafirmar su diferencia y posición socialmente ventajosa.

En este proceso el propio colectivo que busca diferenciarse adopta el discurso racista y estereotipado de la población mayoritaria y de la institución, que les otorga el permiso diferenciándolos públicamente de forma positiva en sus declaraciones. En el caso de Vitoria-Gasteiz, el debate penetró en la sociedad, generando posiciones encontradas y, como consecuencia, conflicto social creando un ambiente de tensión e inseguridad, donde la población está legitimada para sospechar de parte de la sociedad, los magrebíes. Como consecuencia, algunos sectores de la población sienten amenazado su estilo de vida o bienestar social y económico como supuesto resultado de la presencia de ciertos colectivos que identifican como ajenos. En resumen, se ha señalado al otro negativo. Obviamente, este ambiente de crispación tiene consecuencias muy negativas en la convivencia.

También los colectivos "favorecidos" por las declaraciones radicalizan su discurso, incorporando los argumentos y estereotipos como verdades absolutas y desde la posición de "inmigrantes buenos":

Los árabes tienen mucho problema para integrarse con el resto de los inmigrantes. Tienen problemas de integración. Así como yo dominicana puedo salir con una colombiana o peruana o lo que sea, ellos siempre están en su colectivo. LA

Pero porque ellos [los marroquíes] no quieren adaptarse. LA

Yo creo que hace mucho más esfuerzo el gobierno vasco para los árabes que para el resto. Y nosotros sí nos estamos adaptando e integrando. Pero los árabes no. LA

Yo no quiero que den ayudas, porque las ayudas que les están dando a ellos nos perjudican a nosotras. ¿Por qué? Porque esas ayudas no se las dan a las personas mayores que yo cuido y me está quitando el trabajo. Ellos no hacen nada y yo quiero trabajar. $Y$ yo no tengo trabajo. LA

[los magrebies] Viven en no sé dónde, cobran las ayudas, tienen $2.000 €$ de ayudas. $L A$

Pero los moros: pasan seis meses, padrón y ayuda. Y los de Sudamérica trabajan todos, pero con poco dinero. RO

A ver, se está generalizando. Pero yo te digo que lo que escucho no es esto. Todo el mundo habla de eso. LA

Sí, todo el mundo habla de los moros. LA

Como podemos observar en los testimonios, estos argumentos empleados para justificar el racismo guardan mucha relación con lo declarado desde las instituciones. Así, la ausencia de "ganas de integrarse", el esfuerzo innecesario de las instituciones por mejorar la situación del otro negativo, así como el abuso de los bienes generados por el conjunto de la sociedad son argumentos muy 
recurrentes en el discurso de colectivo latinoamericano y, en menor medida, rumano. Al culpabilizar desde las instituciones a un grupo de algo tan relevante como abusar del Estado de Bienestar, se da una ruptura de la cohesión social, se intenta expulsar de la sociedad a todo un colectivo que, sin embargo, continúa compartiendo espacio físico. Es en este punto donde la crispación social genera problemas más o menos graves de convivencia. Cuando el discurso se manifiesta.

Estas consecuencias son percibidas por la propia población inmigrante de estos colectivos estigmatizados, una de las características de la segunda fase de racismo y superiores, que en los grupos de discusión manifestaba la incomodidad, agresiones verbales y la discriminación ejercida por algunos de sus vecinos que se achaca a la tensión social generada desde la institución:

Hay prejuicios pero que se generan desde el ayuntamiento. MO

[...] los comentarios del alcalde tuvieron consecuencias negativas. En una concentración pasaba la gente y nos gritaban “¿cuánto os pagan?". MO

En Vitoria se vive bien pero el problema ha sido a partir de este alcalde que ha marcado diferencias con la gente de fuera. En mi caso, yo creo que antes era mejor, pero desde que ha salido lo del alcalde. $M A$

[...] esta es la principal causa de deterioro de la convivencia porque antes se vivía bien con la gente de Vitoria pero ahora las relaciones se están deteriorando. MA

Siempre he tenido amigas de aquí desde muy pequeña, desde que llegué a Vitoria siempre he salido con sus amigas vascas y vitorianas, nunca ha tenido ningún problema, pero a partir de verano he empezado a discutir con ellas, por las declaraciones del alcalde que salieron en el periódico. Eso se les ha metido en la cabeza, y siento como que me ven mal. MA

[...] la crisis ha tenido sus consecuencias también. Antes se vivía mejor, y ahora con la crisis y los comentarios del alcalde, la gente cree que es verdad lo que decía (el alcalde). $M O$

[...] es bastante complicada la integración por las declaraciones de la alcaldía discriminando a una parte de la población. Para conseguir una integración, es necesario el voto político de los inmigrantes. $M A$

Pienso marcharme por el futuro de mis hijos, pero también por el racismo y discriminación que se generan aquí. En Francia tratan mejor. $M A$

[...] tengo sensación de mala fama que podrían tener los magrebies. $M O$ 
[...] los prejuicios que han generado, si vas bien vestido, la gente empieza a comentar que están bien vestidos gracias a nuestro dinero. $\mathrm{MO}$

Los testimonios de las personas pertenecientes al colectivo señalado por el alcalde no dejan lugar a dudas: la convivencia para ellos ha empeorado notablemente. Manifiestan percibir miradas de recelo, comentarios y, en definitiva, mayor distancia con la población., incluyendo sus entornos más cercanos.

Esta identidad social negativa construida desde todo el conjunto de la sociedad, incluidos los inmigrantes, suscita preocupación en otros colectivos no afectados por la polémica, pero que temen acabar en una situación parecida:

Todos los extranjeros son diferentes. Pero si vienen más y más y uno de nosotros como un marroquí hace cosas malas, caemos todos de malos. $R O$

Pero no sólo se manifiesta a nivel discursivo. Así, el racismo político ha desencadenado una serie de actos y agresiones de corte racista contra los grupos estigmatizados, no sólo por parte de la sociedad receptora sino también apoyada por algunos segmentos de la población inmigrante, tal y como ocurre en el caso que se ha mencionado anteriormente.

En esta misma línea, son destacables dos casos de agresión física ocurridos en noviembre de $2014^{12}$ y febrero de $2015^{13}$, víctimas en ambos casos hombres de origen magrebí. Entre otros sucesos alarmantes podemos mencionar la aparición de pegatinas con esvásticas en varios lugares públicos del municipio, en concreto en el local de una asociación africana, una de las oficinas del servicio vasco de empleo y en la Universidad Pública ${ }^{14}$ (Foto 2). Poco después aparecieron pintadas racistas en una de las mezquitas de Vitoria-Gasteiz (Foto 2). Asimismo, la polémica surgida en torno a un conductor de autobús urbano que no permitió la entrada a una mujer que vestía un velo ${ }^{15}$. Por último, se registraron múltiples agresiones verbales, hecho también mencionado en el grupo de discusión de personas inmigrantes procedentes del Magreb.

${ }^{12}$ Diéz, T. y Muñoz, J (2014, 4 de noviembre).. Una agresión racista termina de envenenar el clima en Gasteiz, Noticias de Navarra.

13 J. C. B. (2015, 5 de febrero). 20 colectivos denuncian una «agresión racista» a un mauritano en Vitoria, EL CORREO.

${ }^{14}$ Gatón, I. (2015, 18 de febrero). Aparecen nuevos mensajes racistas en Lanbide y la Universidad, Gasteiz, hoy.

15 Reyero, I. (2014, 7 de noviembre). Un conductor de autobús urbano de Vitoria prohíbe la entrada a una mujer con velo, $A B C$. 
Foto 2. Pegatinas con mensaje racista y pintadas en la mezquita de Zaramaga, Vitoria-Gasteiz
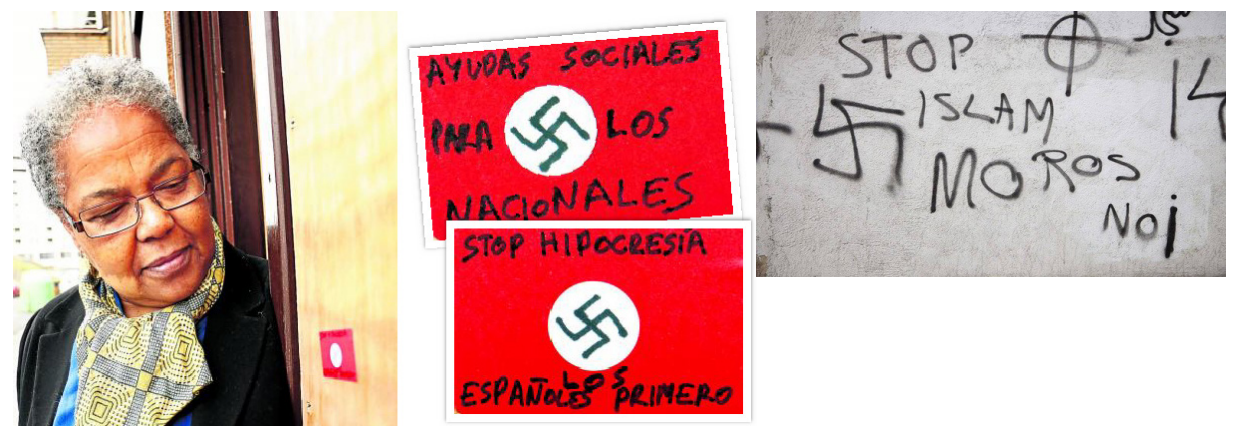

Fuente: Jesús Andrade. EL CORREO ${ }^{16}$ y WEBISLAM.COM ${ }^{17}$

Esta serie de hechos y declaraciones muestran la fragilidad de la convivencia y la cohesión social, que fácilmente pueden verse afectadas por unas declaraciones, especialmente si son formuladas por una figura tan relevante como es la de un alcalde, y más aún cuando quedan legitimados desde los niveles más altos dejando impune este tipo de discursos ${ }^{18}$. Esto reafirma el peligro de sobrepasar la barrera que separa el racismo latente del abierto y violento en sus consecuencias.

A modo de resumen, ofrecemos un esquema que, desde nuestro punto de vista, ayuda a explicar la relación entre el racismo institucional y el despliegue del discurso y actuaciones racistas por propios colectivos de inmigrantes, ya que integra las relaciones entre a éstos últimos en el sistema de creación y reproducción del discruso racista y xenófobo, del que en algunos casos pueden llegar a ser copartícipes.

16 Andradre, J. (2015, 19 de febrero). Abrantes observa una de las pegatinas que aparecieron ayer en la sede de Afro, Vitoria-Gasteiz, Imagen tomada del sitio del periódico EL CORREO: http://www.elcorreo.com/alava/araba/201502/19/ararteko-alerta-sobre-peligro-20150219123312. html. (Fecha de actualización: 4 de mayo de 2017)

17 WebIslam (2016, 12 de noviembre). Acto islamófobo en una mezquita de Vitoria, WebIslam.

18 Recordemos que la denuncia de SOS Racismo Álava contra el alcalde finalmente fue archivada alegando al hecho de que dichas declaraciones se hicieron "en el ejercicio de su libertad a expresar y a difundir libremente los pensamientos, ideas y opiniones mediante la palabra, el escrito o cualquier otro medio de reproducción". http://www.vascopress.com/la-fiscalia-archivala-denuncia-de-sos-racismo-contra-maroto-10032015/ 


\section{Esquema 2. Incidencia del racismo en diferentes capas de la sociedad}

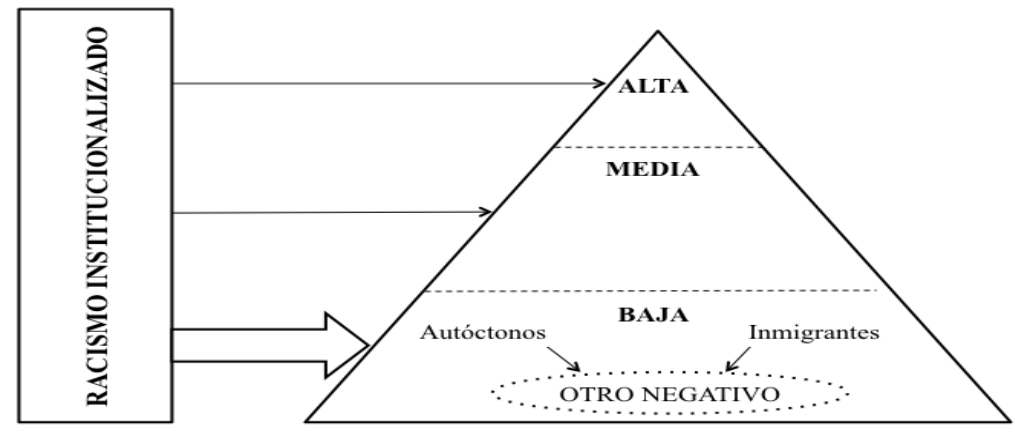

Fuente: Elaboración propia

Así, como se observa en el Esquema 2, la manifestación abierta de las actitudes racistas desde las instituciones públicas afectan a toda la sociedad en su conjunto, pero sobre todo a sus capas más desprotegidas social y económicamente, enfrentando asimismo a las personas autóctonas con las inmigrantes y los diferentes colectivos minoritarios entre sí. El contexto de crisis económica en su momento más intenso y la existencia de un sistema de creencias y actitudes xenófobas y racistas previas en la sociedad, hacen que el racismo político tenga un mayor impacto en el clima de las relaciones sociales y genere un ambiente de tensión y desconfianza. Es en este contexto donde el otro estigmatizado, -la población magrebí en este caso-, se convierte fácilmente en un chivo expiatorio no sólo para la población autóctona, sino también para algunos colectivos de inmigrantes, se suman a estos discursos que conforman las relaciones de poder disperso (Foucault, 1992; Bourdieu, 2001).

En este sentido, cabe señalar que, tal y como afirman muchos estudios (entre otros, Cea y Valles, 2013; Ikuspegi, 2015), el grado de racismo y otras formas de rechazo al otro varía en función de ciertas características sociodemográficas, afectando en mayor medida a las capas más desprotegidas de la estructura social, debido a que la inmigración por lo general se inserta en el estrato más bajo de la sociedad, suponiendo una competencia para la población autóctona con la que lo comparte. Al mismo tiempo, y por esta misma razón, el contacto con esta población es más intenso que el que se da en capas más altas de la estructura social.

Así, al ser más sensibles a cambios sociales, la xenofobia y el racismo impulsados desde arriba (élites políticas) tienen una mayor capacidad de movilización de grupos socialmente vulnerables, sobre todo cuando en el 
imaginario social aparece un enemigo común, un otro negativo que amenaza el orden social existente, infringiendo las normas y, en palabras de Schütz, cuestionando "el sistema de recetas verificadas disponible" (Schütz, 1964: 99).

\section{CONCLUSIONES}

Las actitudes racistas, ya sean procedentes del colectivo mayoritario o dentro del propio colectivo minoritario, no surgen de manera espontánea. Cómo casi todo en el ámbito social, la formación de actitudes colectivas conlleva un proceso en el que dichas actitudes se manifiestan de diversas formas y en distintas intensidades. En este sentido, partimos de la hipótesis de que los inmigrantes no son meros perceptores del racismo, sino también pueden convertirse en sus emisores. Son, por tanto, actores activos en todo el proceso de la construcción de otredad, que se les transfiere desde la población mayoritaria y que retransmiten a otros segmentos de la sociedad más vulnerables, al otro -u otros- negativo/s. Esto se manifiesta sobre todo a medida que los niveles del racismo general van subiendo e intensificándose, como ocurrió en el caso que se ha cogido de ejemplo.

En el modelo teórico elaborado en este trabajo y basado en las aportaciones de diversos autores/as, se establecen cuatro fases dentro de este proceso en las que el posicionamiento institucional establece un punto de inflexión. Podemos identificar claramente elementos de dichas fases en la ciudad de Vitoria-Gasteiz en el año 2014, en el que se realizaron declaraciones racistas y xenófobas por parte de las élites políticas del municipio.

En relación a las fases, el racismo comienza con la estigmatización de un colectivo, la caracterización del otro negativo que se percibe como ajeno y diferente, un elemento que destruye la soñada homogeneidad ordenada y que amenaza con la anomia al portar culturas diferentes. Esta es la fase del discurso estereotipado que no llega a la acción. En esta fase observamos ya un colectivo que destaca por excesiva y negativa categorización, tanto por parte de la población autóctona como por la propia población inmigrante: el colectivo magrebí. No se trata únicamente de una tendencia local, la islamofobia es un fenómeno internacional que atribuye características de violencia, machismo y barbarie tanto a la confesión como a todas aquellas personas que se identifican con ella.

Como se muestra en el presente trabajo, estos discursos se vuelven visibles o tangibles al tomar cuerpo en el espacio urbano en forma de concentración espacial, acompañado de un clima de tensión en el que la figura del otro negativo se intensifica generando un cierto aislamiento del mismo. En nuestro caso cabe destacar que dicho aislamiento no se lleva a cabo únicamente por parte de la población autóctona. Las declaraciones de las personas inmigrantes de otros colectivos evidencian el deseo de no compartir espacio físico o interacción con determinados colectivos "malos". La estigmatización surte efecto y tiene consecuencias reales y palpables. 
Hasta el momento, las instituciones públicas se mantienen "al margen" de estos procesos sociales, sin embargo, el papel de éstas supone la diferencia entre los que se podría denominar un estado latente y uno manifiesto. En otras palabras, se trata de situaciones en las que las instituciones se apropian del discurso social mayoritario y lo apoyan.

Este apoyo o legitimación del discurso racista y xenófobo dirigido al colectivo más estigmatizado (el magrebí), que se pudo observar recientemente en Vitoria-Gasteiz, suscita un rechazo hacia los grupos acusados no sólo en la sociedad receptora, sino también entre algunos colectivos de la población inmigrante, que han optado por sumarse a la nueva norma social adoptando los discursos más radicales de la población autóctona. Esta división de los inmigrantes en "buenos" y "malos" ha convertido a los grupos peor parados en el chivo expiatorio hacia el que dirigir la frustración, y ha radicalizado a los grupos minoritarios "privilegiados" en la expresión del rechazo. Esta cadena de hechos muestra la fuerza y la relevancia de las instituciones y líderes políticos en la formación de la opinión pública y remarca su responsabilidad para la convivencia. Nos muestra a su vez el peligro de las actitudes racistas y xenófobas, especialmente cuando cuentan con apoyo institucional, aunque sea puntal, es lo que se denomina racismo institucionalizado y/o político.

$\mathrm{Y}$ es que el racismo no afecta de igual manera a todo el mundo. En el contexto actual, al hablar de racismo debemos hacerlo también de estructura social. Las capas más desprotegidas de la estructura social lo sufren en mayor medida, generándose incluso nuevas categorías dentro de las bajas capas que convierten a los colectivos más "privilegiados" en actores activos del racismo. El racismo se manifiesta como instrumento diferenciador con el que poder mantenerse en una posición más elevada.

Es difícil concluir en qué fase de este proceso nos encontramos. Hablar de una situación de racismo institucionalizado sistemático sería exagerar una situación puntual y condenada por el resto de formaciones políticas. Sin embargo, sí merece la pena resaltar las repercusiones sociales de unas simples declaraciones. Un contexto plagado de estereotipos que por sí mimo construye la imagen del otro negativo tiene alas al obtener legitimación institucional. Las consecuencias para la cohesión social son nefastas y de lenta recuperación y, es por este motivo que, las instituciones deben asumir su responsabilidad y actuar como elementos de contención elaborando políticas públicas que favorezcan la convivencia y mostrando su rechazo público hacia este tipo de actitudes $\mathrm{y}$, nunca, como catalizador de las actitudes racistas y xenófobas. 


\section{BIBLIOGRAFÍA}

ALLPORT, G. (1962): La naturaleza del prejuicio, Buenos Aires, Eudeba.

ANDRADRE, J. (2015, 19 de febrero): "Abrantes observa una de las pegatinas que aparecieron ayer en la sede de Afro", Vitoria-Gasteiz, Imagen tomada del sitio del periódico EL CORREO: http://www.elcorreo.com/alava/araba/201502/19/arartekoalerta-sobre-peligro-20150219123312.html. [consulta: 04-05-2017]

BAKER, M. (1981): The New Racism, London, Junction Books.

BECK, U. (2000): La democracia y sus enemigos, Barcelona, Paidós.

BOURDIEU, P. (2001): Poder, derecho y clases sociales, Bilbao, Descelée de Brouwer. BOURDIEU, P. \& WACQUANT, L.J.D. (1992): Réponses. Pour une anthropologie reflexive, Paris, Seuil.

CARMICHAEL, S. \& HAMILTON, C. V. (1967): Black Power: the Politics of Liberation in America, Nueva York, Vintage Books.

CEA D'ANCONA, M. A., VALLES, M., Y ESEVERRI, C. (2013): Inmigración: filias $y$ fobias en tiempos de crisis, Madrid, Biblioteca Nueva.

CEA D’ANCONA, M. A. (2009): "La compleja detección del racismo y la xenofobia a través de encuesta: Un paso adelante en su medición", Revista Española de Investigaciones Sociológicas, 125, pp. 13-45.

CEA D’ANCONA, M. A. Y VALLES MARTÍNEZ, M. S. (2013): Evolución del racismo, la xenofobia y otras formas conexas de intolerancia en España [Informe 2013], Madrid, OBERAXE, Ministerio de Trabajo e Inmigración.

CEA D’ANCONA, M. A. Y VALLES MARTÍNEZ, M. S. (2014): Evolución del racismo, la xenofobia y otras formas conexas de intolerancia en España, [Informe 2014], Madrid, OBERAXE, Ministerio de Empleo y Seguridad Social.

CHEN, C. (2010): Minority on minority discrimination: Impact of majority social norm perception [Technical report], Carnegie Mellon University.

CUADRADO, I, LÓPEZ- RODRIGUEZ, L, NAVAS, M. (2016): "Las perspectivas de la minoría: estereotipos y emociones entre grupos inmigrantes", Anales de Psicología, 32 (2), disponible en http://dx.doi.org/10.6018/ analesps.32.2.205341 [consulta: 04-05-2017]

D'APPOLLONIA, A.CH. (1988): Los racismos cotidianos, Barcelona, Bellaterra.

DIÉZ, T. \& MUÑOZ, J. (2014, 4 de noviembre): "Una agresión racista termina de envenenar el clima en Gasteiz", Noticias de Navarra, disponible en: http://www. noticiasdenavarra.com/2014/11/05/sociedad/euskadi/una-agresion-racista-terminade-envenenar-el-clima-en-gasteiz [consulta: 04-05-2017]

DOUGLAS, M. (1973): Pureza y peligro: Un análisis de los conceptos de contaminación y tabú, Madrid, Siglo XXI.

FERNÁNDEZ, J. (2005): "La noción de violencia simbólica en la obra de Pierre Bourdieu: una aproximación crítica”, Cuadernos De Trabajo Social, 18, pp. 7-31.

FOUCAULT, M. (1992): Genealogía del racismo, Madrid, Siglo XXI de España Editores.

GABINETE DE PROSPECCIÓN SOCIOLÓGICA (2012): El Estado del Bienestar. Dossier de prensa, Gobierno Vasco, disponible en: https://www.irekia.euskadi. eus/uploads/attachments/2313/estado bienestar dossier es .pdf?1340270907 [consulta: 04-05-2017]

GAERTNER, S. L. Y DOVIDIO, J. F. (1986): “The aversive form of racism” en J. F. Dovidio y S.L. Gaertner (Eds.) Prejudice, discrimination and racism, San Diego, Academic Press, pp. 35-59. 
GATÓN, I. (2015, 18 de febrero): “Aparecen nuevos mensajes racistas en Lanbide y la Universidad", Gasteiz hoy, disponible en: http://www.gasteizhoy.com/aparecenmensajes-nazis-y-racistas-en-el-local-de-la-asociacion-afroamericana/ [consulta: 04-05-2017]

GIMENO GIMÉNEZ, L. (2001): Relación entre las investigaciones cualitativas y cuantitativas, Madrid, CIS.

IKUSPEGI (2015): Barómetro 2015. Percepciones y actitudes hacia la inmigración extranjera, Bilbao, Ikuspegi.

IKUSPEGI (2014): Estudio diagnóstico sobre el fenómeno migratorio en VitoriaGasteiz, disponible en http://www.ikuspegi.eus/documentos/informes/estudio migracion vitoria-gasteiz.pdf [consulta: 04-05-2017]

INE. Instituto Nacional de Estadística. http://www.ine.es

IZAOLA, A. Y ZUBERO, I. (2015): "La cuestión del otro: forasteros, extranjeros, extraños y monstruos", Papers, 100/1, pp. 105-129.

J. C. B. (2015, 5 de febrero): "20 colectivos denuncian una «agresión racista» a un mauritano en Vitoria", EL CORREO, disponible en: http://www.elcorreo.com/ alava/araba/201502/05/colectivos-denuncian-agresion-racista-20150204222819. html [consulta: 04-05-2017]

JONES, J. M. (1972): Prejudice and racism, Reading, MA, Addison Wesley Publishers.

KINDER, D. R \& SEARS, D. O. (1981): "Prejudice and politics: Symbolic racism versus racial threats to the good life", Journal of Personality and Social Psychology, 40, pp. 414-431.

KINDER, D. R. Y SEARS, D. O. (1981): "Prejudice and politics: Symbolic racism versus racial threats to the good life", Journal of Personality and Social Psychology, 40, pp. 414-431.

MCCONAHAY, J.B. (1986): "Modern racism, ambivalence, and the modern racism scale" en J.F. Dovidio, y S.L. Gaertner (Eds.) Prejudice, Discrimination and Racism, Orlando, Academic Press, pp. 91-126.

MCCONAHAY, J.B. (1986): "Modern racism, ambivalence, and the modern racism scale", Prejudice, Discrimination and Racism, pp. 91-126.

PARK, R. E., BURGUESS, E. W. \& MCKENZIE, R. (1967): “The city”, The university of Chicago Press, pp. 1-46.

PETTIGREW, T. Y MEERTENS, R. W. (1995): "Subtle and blatant prejudice in Western Europe", European Journal of Social Psychology, 25, pp. 57-75.

REYERO, I. (2014, 7 de noviembre): "Un conductor de autobús urbano de Vitoria prohíbe la entrada a una mujer con velo", $\mathrm{ABC}$, disponible en: http://www.abc.es/ espana/20141107/abci-autobusero-prohibe-velo-201411071311.html [consulta: 0405-2017]

SCHÜTZ, A. (1974): "El forastero", en Estudios sobre teoría social, Buenos Aires, Amorrortu, pp. 95-107.

SHAPIRO, J. R., Y NEUBERG, S. L. (2008): "When do the stigmatized stigmatize? The ironic effects of being accountable to (perceived) majority group prejudiceexpression norms", Journal of Personality and Social Psychology, 95, pp. 877-898.

SHERIF, M. (1966): In common predicament: Social psychology of intergroup conflict and cooperation, Boston, Houghton Mifflin.

SIMMEL, G. (1977): "Sociología: Estudios sobre las formas de socialización”, Tomo 2, Revista de Occidente.

TAGUIEFF, P. (1988): La Force du préjugé. Essai sur le racisme et ses doubles, Paris, La Decouverte. 
TAJFEL, H. (1981): Human groups and social categories: Studies in social psychology, Cambridge, Cambridge University Press.

TAJFEL, H. Y TURNER, J.C. (1986): “The social identity theory of intergroup behavior", en G. Austin y S. Worchel (eds.) Psychology of intergroup relations, Chicago, Nelson Hall, pp. 7- 24.

WIEVIORKA, M. (1992): El espacio del racismo, Barcelona, Paidós.

WIEVIORKA, M. (2009): El racismo: una introducción, Buenos Aires, Gedisa. 